Résumés des conférences et travaux

\title{
Démotique
}

\section{Michel Chauveau}

\section{(2) OpenEdition Journals}

Édition électronique

URL : https://journals.openedition.org/ashp/3436

DOI : $10.4000 /$ ashp.3436

ISSN : 1969-6310

Éditeur

Publications de l'École Pratique des Hautes Études

Édition imprimée

Date de publication : 1 septembre 2020

Pagination : 4-10

ISSN : 0766-0677

Référence électronique

Michel Chauveau, « Démotique », Annuaire de l'École pratique des hautes études (EPHE), Section des sciences historiques et philologiques [En ligne], 151 | 2020, mis en ligne le 09 juillet 2020, consulté le 06 juillet 2021. URL : http://journals.openedition.org/ashp/3436 ; DOI : https://doi.org/10.4000/ashp. 3436 


\section{DÉMOTIQUE}

\section{Directeur d'études : M. Michel Chauveau}

Programme de l'année 2018-2019 : I. Textes documentaires. — II. Papyrus littéraires.

Le directeur d'études a présenté aux auditeurs trois papyrus démotiques inédits d'époque saïte, ayant appartenu à une ancienne collection parisienne, et acquis récemment par la fondation Carlsberg à Copenhague ${ }^{1}$. Ces documents proviennent d'archives de choachytes thébains et comprennent deux contrats établissant une association pour l'entretien d'une tombe, ainsi qu'un reçu qui a particulièrement retenu l'attention des auditeurs et dont voici la translittération accompagnée d'une traduction :

pCarlsberg 944

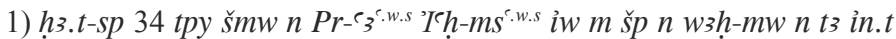

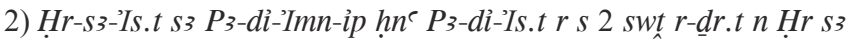

3) N3-mnh-hpr-Re 'Iy-m-htp sz N3-mnh-hpr-Re dỉ=k mtì ḩз.t

4) =n nз ${ }^{\top} k(. w) r-\underline{d b} b_{3} n_{3} w n-p r . w n$ s.hm.t Ta-p3-'ty.t ta 'Ir.t=w-rd t3-

5) $y=t n m w . t \check{s} p=n$ s.t ḩ3.t=n mty.w n.im=w ỉwty $s p$

6) sh 'Imn-ḥtp s3 N3-mnh-hpr-Re

An 34, mois de Pachons, du roi Amasis. Bon comme reçu pour le choachyte de la Vallée Harsiésé fils de Pétéamenopé, ainsi que pour Pétéisé, ce qui fait deux personnes, délivré de la part d'Hor fils de Mencheperrê (et) d'Imhotep fils de Mencheperrê : « Tu (sic) as contenté nos cœurs des rations en rétribution des ouvertures-de-maison pour la dame Tapaty fille de Iretouredj, votre mère. Nous les avons (bien) reçues et nos cœurs en sont satisfaits, sans (qu'il y ait de) reste. » Écrit par Amenhotep fils de Mencheperrê. Suivent les signatures de deux témoins.

Ce reçu, d'un type formel bien connu à Thèbes à l'époque saïte ${ }^{2}$, est remarquable

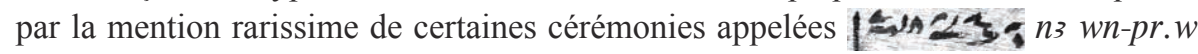
« les ouvertures de maison(s) ». Celles-ci, accomplies soit à l'occasion des fêtes en l'honneur d'Osiris au mois de Khoiak ${ }^{3}$, soit lors du processus des funérailles d'un particulier, ne sont attestées que par un petit nombre de textes funéraires ou religieux d'époque tardive. Ainsi dans le rituel du papyrus Leyde T 32, V, 27-28: « On dirige pour toi tout le cérémonial de la ouâbet les jours de $w n-p r »{ }^{4}$. Cette pratique est

1. Rapport préliminaire publié par I. Adsbøl Christensen et K. Ryholt, dans Dansk Eegyptologisk Selskab, Papyrus, 39, n 1, Copenhague, 2019, p. 16-23.

2. Cf. K. Donker van Heel, Abnormal Hieratic and Early Demotic Texts collected by the Theban Choachytes in the Reign of Amasis, Leyde, 1995, doc. 3, 12 et 14-16.

3. E. Chassinat, Le mystère d'Osiris au mois de Khoiak, t. 2, Le Caire, 1968, p. 635-636.

4. F. Herbin, Le livre de parcourir l'éternité, Louvain, 1994 (OLA 58), p. 213. 
quelque peu explicitée dans le papyrus digraphe hiératico-démotique Rhind I qui ne dénombre pas moins de 17 wn-pr.w qui doivent être effectués durant les 70 jours de l'embaumement ${ }^{5}$. Le reçu cité ci-dessus doit en conséquence concerner l'enterrement de la mère supposée défunte du choachyte Harsiésé et de Pétéisé, frère utérin du premier. Il paraît cependant singulier qu'un choachyte, normalement compétent en cette matière, ait dû recourir à des étrangers pour l'accomplissement de ces rites liés aux funérailles de sa propre mère. L'absence de tout titre justifiant l'intervention des bénéficiaires des rations en question est également déconcertante. Bien qu'il soit généralement considéré que ces wn-pr.w consistaient en des processions, le sens même de l'expression semble impliquer qu'il s'agissait avant tout de permettre l'accès à une enceinte sacralisée. Dans un contexte funéraire, cette dernière ne devait être autre que la ouâbet où la dépouille du défunt subissait le long processus d'embaumement. De toute évidence, l'ouverture d'un tel lieu n'entrait pas dans les compétences du choachyte qui devait faire appel à des personnes autorisées par leurs fonctions spécifiques. On songe alors naturellement aux «pastophores », dont la désignation égyptienne de $w n-p r$, « ouvreur de maison », correspondrait très exactement au nom des cérémonies mentionnées dans notre reçu, expliquant ainsi son omission par le scribe pour qualifier les exécutants d'un tel rite. Or, il a déjà été remarqué que les choachytes thébains, à partir du règne de Darius, portent également le titre de "pastophores d'Amon de Djémé », qualification qu'ils ne semblent pas avoir affichée auparavant ${ }^{6}$. Une telle expansion de leur titulature s'expliquerait dès lors aisément : puisqu'à l'époque de notre reçu, une part non négligeable du processus funéraire liée aux fonctions des pastophores leur échappait, ainsi que les revenus afférents, on peut supposer que les choachytes thébains aient tenu d'une manière ou d'une autre à intégrer cette corporation, ce qu'ils n'ont pu apparemment accomplir qu'entre la fin du règne d'Amasis et l'an 5 du règne de Darius (517 av. J.-C.) où l'acquisition de ce titre par des choachytes est pour la première fois attestée ${ }^{7}$.

Cette déduction logique se heurte toutefois à la lecture quasi unanimement adoptée aujourd'hui pour la version démotique du titre "pastophore » que nous livrent les textes grecs. Ce qu'on lisait depuis le début du siècle dernier $w n$ ou wn-pr est maintenant translittéré iry-`3, suivant un remarquable article paru en 2014 et dû à F. Hoffmann et à J. F. Quack ${ }^{8}$. Dans une contribution de près de trente pages, ces deux auteurs ont minutieusement examiné les données de ce problème complexe. En effet, bien que la transcription hiéroglyphique de la graphie démotique

5. G. Möller, Die beiden Totenpapyrus Rhind, Demotische Studien, 6, Leipzig, 1913, p. 78, n. 41. Ces rites sont également mentionnés dans un compte thébain pour des dépenses funéraires écrit en hiératique anormal, P. Louvre E $3228 \mathrm{H}$, où il est question d'un paiement pour de la résine utilisée dans deux séries de dix wn-pr (en cours de publication par K. Donker van Heel qui en a donné une présentation préliminaire dans JEA 101, 2015, p. 320-325).

6. Cf. S. P. Vleeming, Hundred-Gated Thebes (P. L. Bat. 27), Leyde, 1995, p. 243.

7. pTsenhor 4, 1.5; cf. P. W. Pestman, Les papyrus démotiques de Tsenhor, Louvain, 1994, p. 51 : « Mes charges de choachyte et de pastophore dans la nécropole ».

8. «Pastophoros », dans “A Good Scribe and Exceedingly Wise Man” Studies in Honour of W. J. Tait (A. Dodson, J. Johnston et W. Monkhouse éd.), Londres, 2014 (GHP Egyptology 21), p. 127-155. 
titre en question ne puisse être mise en doute : 9 , sa lecture exacte n'a jamais été évidente. Le premier groupe composé d'un vantail de porte et d'un bras armé fut interprété par W. Spiegelberg en 1901 comme une graphie du verbe wn « ouvrir », privée des éléments phonétiques initiaux é réduite aux seuls déterminatifs ${ }^{10}$. Ce pionnier des études démotiques se fondait sur des inscriptions en hiératique de vases découverts lors des fouilles menées par le marquis de Northampton dans la nécropole thébaine à la fin du XIX ${ }^{\mathrm{e}}$ siècle ${ }^{11}$. Quelques unes d'entre elles mentionnaient un wn-pr $m$ imnt, «Pastophore in the West», bien pourvu du groupe phonétique 12 , mais il n'était pas clair si ces mentions concernaient des officiants chargés d'accomplir des rites funéraires, c'est-à-dire probablement les choachytes eux-mêmes, ou bien plutôt les rites processionnels connus par le pRhind I et le T 32 de Leyde, cette dernière interprétation étant la plus probable.

Bien que la lecture wn ou wn-pr fût malgré tout admise par l'ensemble des égyptologues durant tout le $\mathrm{xx}^{\mathrm{e}}$ siècle, la similitude graphique entre la transcription en hiéroglyphes du lexème démotique iry-c 3 믄 " préposé à la porte », ainsi que la proximité de sens et d'emploi des deux fonctions, ont conduit les auteurs précités à faire la démonstration de leur identité parfaite sous ce dernier vocable, en rejetant définitivement la translittération $w n(-p r) \mathrm{du}$ titre démotique avancée par Spiegelberg.

Malgré le consensus récent de la communauté démotisante en faveur de cette nouvelle lecture iry-`3, et sans intention polémique particulière, l'hypothèse évoquée ci-dessus concernant la signification du P. Carlsberg 944 nous a incité à procéder à un examen approfondi de la question lors de plusieurs séances consécutives consacrées à ce problème.

La lecture du titre démotique correspondant au " pastophore ». - Par commodité et pour éviter toute confusion, nous désignerons le groupe démotique en discussion par le sigle VDP (= version démotique du pastophore). Il nous semble que la pierre angulaire de la démonstration en faveur de VDP = iry- ${ }_{3}$ a été la publication d'un onomasticon démotique, le pCarlsberg 425, due à K.-T. Zauzich ${ }^{13}$. Cette liste disposée en colonnes comporte essentiellement des anthroponymes classés selon leur lettre initiale dans l'ordre alphabétique sudarabique. À la fin de la section affectée à la lettre $I$, on trouve trois titres ( $\left.\mathrm{n}^{\text {os }} 151-153\right)$ apparemment formés sur VDP : VDP (n) $\ulcorner$ ? ; VDP

9. Celle-ci est prouvée par la plus ancienne occurrence de ce titre démotique, celle du pRylands 6 (an 3 d'Amasis), dont la graphie est encore très proche du hiératique : מע.

10. W. Spiegelberg, «Paapis », Recueil de Travaux relatifs à la philologie et à l'archéologie égyptiennes et assyriennes, 23 (1901), p. 98-99.

11. W. G. of Northampton, W. Spiegelberg et P. E. Newberry, Report on some Excavations in the Theban Necropolis during the Winter of 1898-9, Londres, 1908, p. 24 et pl. XXXII.

12. À noter toutefois que, dans l'une de ces inscriptions ( $\left.\mathrm{n}^{\circ} 7\right)$, la graphie $w n$ est réduite aux seuls déterminatifs, ce qui la rend identique à la graphie démotique du titre de pastophore.

13. «Ein antikes demotisches Namenbuch », dans P. J. Frandsen et K. Ryholt éd., A Miscellany of Demotic Texts and Studies), Copenhague, 2000 (CNI Publ. 22, The Carlsberg Papyri 3), p. 27-52. Le papyrus daterait du $\mathrm{IV}^{\mathrm{e}}$ siècle avant notre ère par la paléographie, cf. loc. cit., p. 33-34. 
$n$ pr; VDP (n) $P r$ - 3 . Puisque l'initiale 'I excluait a priori la lecture $w n-p r$, VDP fut lu iry- 3 par l'éditeur qui y vit la confirmation d'une hypothèse déjà émise par Joachim Quack, et que ce dernier put ensuite développer dans l'article publié en 2014 avec Friedhelm Hoffmann ${ }^{14}$. Cependant, le troisième titre $\left(n^{\circ} 153\right)$, le seul qui soit de lecture et de sens absolument clairs, semblerait révéler l'existence d'un «VDP royal », ce qui suscite une certaine perplexité, puisque l'ensemble de la documentation démotique datée entre la dynastie saïte et l'époque romaine n'atteste le titre VDP que dans le seul domaine strictement religieux. De fait, un unique parallèle connu d'un VDP lié au roi se trouve dans un autre onomasticon, d'époque romaine et provenant de Tebtynis, le pCarlsberg 23, publié en 1984 par W. J. Tait ${ }^{15}$. Cette liste répertorie cette fois uniquement des titres regroupés par affinité sémantique. Parmi ceux-ci semblait figurer un VDP (n) $\mathrm{Pr}_{-} \mathrm{C}_{3}$, translittéré avec hésitation $w n-\mathrm{pr}-\mathrm{Pr}-\mathrm{C}_{3}$ par l'éditeur et traduit par «Pastophorus of Pharaoh ». Hoffmann et Quack ont effectivement relevé l'étrangeté de l'expression iry- 3 Pr-`3, en évoquant la possibilité d'une haplographie du signe de la maison, ce qui permettrait de lier de manière plus appropriée la fonc-

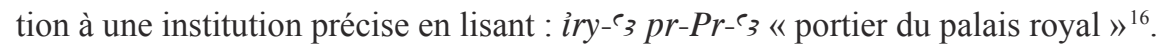

Cependant, s'il faut bien admettre la lecture $i r y-{ }_{3}$ dans ces deux papyrus, puisque le classement alphabétique de l'onomasticon pCarlsberg 425 l'impose, il n'est nullement évident que les titres livrés dans ces listes à but didactique soient réellement formés avec l'élément VDP, et que la translittération de ce dernier doive être également iry- C $_{3}$ dans tous les cas. En effet, l'identification du groupe graphique VDP dans ces occurrences tient uniquement au signe de la maison que les éditeurs ont interprété comme étant le déterminatif obligatoirement inclus dans ce groupe. Mais rien n'impose une telle inclusion : au contraire en dissociant le signe de la maison de l'élément initial, les problèmes interprétatifs posés par ces titres peuvent être aisément résolus. On obtient ainsi, en accord avec la suggestion de Hoffmann et Quack,

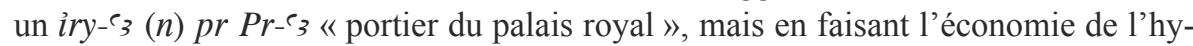
pothèse d'une haplographie ${ }^{17}$. Pour le pCarlsberg $425 \mathrm{n}^{0} 152$, le iry- 3 $n \mathrm{pr}$ «portier de maison » lu par Zauzich, désignation dont le sens serait étrangement vague et indéfini, deviendra sans difficulté un iry-c 3 (n) pr-ha ( trésor ». Enfin, on pourra même proposer pour la séquence partiellement lacunaire au no 151 : iry-`3 (n) pr- $\ulcorner n s w\urcorner$ ( un ensemble de variations parfaitement cohérentes pour un titre que les auteurs de

14. La preuve fournie par le pCarlsberg 425 n'est cependant évoquée qu'à la p. 141 de l'article de Hoffmann et Quack.

15. J. Tait, «A Demotic List of Temple and Court Occupations: P. Carlsberg 23 », H.-J. Thissen et K.-T. Zauzich éd., Grammata Demotika. Festschrift für Erich Lüddeckens, Würzburg, 1984, p. 211-233.

16. Hoffmann et Quack, p. 145, n. 155.

17. J. Tait, op. cit. p. 218, a d'autre part signalé que le signe de la maison surmonté d'un trait horizontal permettrait dans le pCarlsberg 23 de distinguer la graphie du mot $p r$ du simple déterminatif. On peut

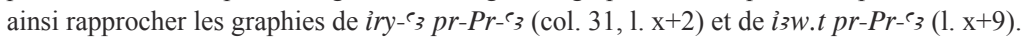

18. En restituant le signe du pavois divin dans la lacune. La graphie $p r-n s w$ doit être considérée comme une abréviation de $p r$-ipy-nsw « harem royal », cf. K. Ryholt, The Petese Stories II, Copenhague, 2006 (CNI Publ. 29, The Carlsberg Papyri 6), p. 36. 
ces onomastica devaient considérer comme essentiellement profane, par opposition à VDP, lié quant à lui invariablement au personnel des temples. La graphie démotique du titre $i r y-c_{3}$ correspondrait alors très exactement à la graphie hiéroglyphique abrégée connue pour ce titre: serait plus à justifier ${ }^{19}$. Considéré ainsi, le pCarlsberg 425, loin de prouver la lecture iry- ? $_{3}$ pour VDP, fournirait au contraire le meilleur argument pour distinguer iry- ${ }_{3}$ de VDP. Il faut d'ailleurs remarquer que l'introduction d'éléments inusités dans ces onomastica devait constituer l'une des priorités didactiques de leurs auteurs, et par voie de conséquence une source de grandes difficultés pour leurs modernes éditeurs. Le compilateur du pCarlsberg 425 a probablement placé à dessein ces trois titres à la fin de la section consacrée à la lettre $I$, tous trois liés à des institutions laïques, afin d'attirer l'attention des jeunes scribes sur leur véritable lecture et leur différence avec le titre VDP auquel ils étaient infiniment plus accoutumés ${ }^{20}$. Quant au pCarlsberg 23, la mention d'un simple portier du palais, au lieu d'un «pastophore royal », permettrait d'éclairer la nature de l'onomasticon dans lequel on ne trouverait plus de section consacrée à des « Temple occupations ", mais où figurerait en revanche une liste d'emplois subalternes liés au palais (parmi lesquels seraient cités, outre le portier, un barbier et des fabricants de perles, de tentes et ornements divers), par opposition aux dignités auliques inventoriées plus bas dans une nouvelle section intitulée $33 w . t$ (n) pr-Pr- 3 « offices du palais royal ».

Quant à la lecture de VDP pour laquelle il nous semble nécessaire d'envisager de nouveau wn-pr, elle a été à notre sens assez solidement établie en 1956 par H. De Meulenaere $^{21}$, en dépit des critiques quelque peu spécieuses de Hoffmann et Quack. Les documents hiéroglyphiques et l'inscription digraphe hiéroglyphique / démotique mis en perspective par De Meulenaere devraient suffire à s'en convaincre. La stèle digraphe Carlsberg ÆIN 636 assure sans aucun doute possible l'équivalence entre VDP et le titre hiéroglyphique $\frac{1}{\square}$, ce dernier groupe étant nettement distinct de La table d'offrandes du Caire CG 23160 présente d'autre part en parallèle les titres VDP $n$ Min et

19. Voir Hoffmann et Quack, p. 140, qui peinent à justifier la disparité entre les graphies hiéroglyphiques et démotiques supposées de iry- 3 sur ce point. L'argument avancé qu'il s'agirait d'un discriminant permettant de distinguer ce titre d'autres groupes homographes en démotique n'est pas convaincant, puisque la plus ancienne attestation démotique connue (pRyl. 6; cf. supra n. 9), proche d'une graphie hiératique, est déjà pourvue du signe de la maison alors même qu'il n'existait alors aucun groupe homographe avec lequel une confusion aurait été possible.

20. À noter que le $\mathrm{n}^{\circ} 103 \mathrm{du}$ pCarlsberg 425 , dans la section des noms à lettre initiale $P$, et translittéré $P_{3}$-iry- ${ }_{3}$ par Zauzich, doit en revanche bien être lu $P_{3}$-VDP, c'est-à-dire selon nous $P_{3}$-wn-pr ! L'auteur de l'onomasticon s'est visiblement plu à glisser cette difficulté homographique afin d'exercer la sagacité de ses lecteurs...

21. H. De Meulenaere, « Pastophores et Gardiens des Portes », CdÉ, 31 (1956), p. 299-302.

22. D'après la photo publiée dans le Catalogue du Caire, le signe qui suit wn est bien (c) et non qui figure par erreur une fois chez De Meulenaere, op. cit., p. 301 (paraissant curieusement démentir sa note 2, mais la version correcte figure en bas de la même page). Même erreur dans la publication de A. Awadalla, Studies in Honor of Ali Radwan (Suppl. ASAE 34), vol. 1, Le Caire, 2005, p. 159; ainsi que chez Hoffmann et Quack, p. 133 et 138. 
arguments de Hoffmann et Quack qui tiennent à dissocier ces deux derniers titres en considérant que le second serait une variante d'un titre sacerdotal attesté essentiellement à Thèbes wn `.wy (n) p.t « ouvreur des portes du ciel». La provenance akhmimique des deux documents, l'absence du duel `.wy ainsi que celle de la mention du « ciel » sont en défaveur d'une telle identification. En revanche, la disposition même des textes hiéroglyphiques sur la table d'offrandes permet de justifier l'expansion du titre du dédicataire, le lapicide ayant disposé d'un bien plus large espace à remplir sur la bordure droite de la table qu'au dessus de la représentation du défunt au centre. Il faut aussi remarquer que, dans chacune des deux déclinaisons d'identité, le père porte le même titre naere qui identifie ce dernier, lu wn-pr n Min «ouvreur de maison de Min », avec $w n{ }^{-}$¿ $n$ pr Min « ouvreur de porte de la maison de Min ». Enfin, il faut ajouter que le titre $\int_{0}^{0} h r y w n-p r$ 'Is.t « chef des pastophores d'Isis », copié par De Meulenaere sur un cartonnage de momie du musée d'Assouan, et que Hoffmann et Quack mettent en doute car ils n'ont pas pu en vérifier eux-mêmes la lecture d'après l'original apparemment égaré, paraît également attesté en démotique dans le dipinto du temple ptolémaïque d'Isis à Assouan no 8 qui mentionne un ḥry VDP $n$ 'Is. $t^{23}$.

En outre, la publication toute récente d'un papyrus funéraire hiératique ${ }^{24}$, probablement d'origine thébaine et daté de la fin du IV ${ }^{\mathrm{e}}$ siècle avant notre ère, semble enfin apporter une preuve définitive de la lecture $w n-(p r)$ pour VDP. Le défunt, $h r i ̉=s-n=f$,

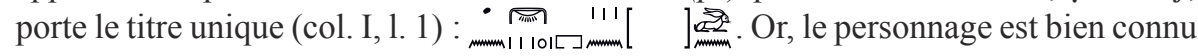
dans la documentation démotique, notamment dans le P. Louvre E 2439, 1. 2, daté de 330 av. J.-C., où le titre correspondant est VDP $n$ pr nbw $n$ 'Imn. En suivant l'éditrice de ce papyrus funéraire ${ }^{25}$, il n'est guère douteux que le titre hiératique en partie lacunaire doive être ainsi complété : ${ }^{(1.1)} w n-\left[p r\right.$ ?]. $w n$ pr $n b w n^{(1.2)}$ [Imn]. Quelle que soit l'incertitude sur la restitution du signe $p r$, il est en tout cas évident que VDP doit comporter l'élément $w n$, confirmant ainsi ce que les documents hiéroglyphiques réunis par De Meulenaere permettaient d'établir.

Pour résumer, nous croyons que les titres iry- $\varsigma_{3}$ 《 portier » et $w n-p r$ « pastophore » ne doivent pas être confondus et que leurs graphies respectives, malgré d'évidentes similitudes, restent parfaitement distinctes. Le signe de la maison, bien plus qu'un simple déterminatif, est un élément constitutif indispensable de $w n-p r^{26}$, tandis qu'il est constamment absent des graphies abrégées de iry-`3, que celles-ci soient hiéroglyphiques ${ }^{27}$ ou (exceptionnellement) démotiques :

23. E. Bresciani, Assuan, Pise, 1978, p. 127 (daté de l'an 5 de Tibère).

24. Ann-Katrin Gill, "The Glorifications of Herisenef in the Museo Egizio (P. Turin Cat. 2117-R 08)», Rivista del Museo Egizio, 3 (2019), Turin, p. 1-21.

25. Op. cit., p. 3 et notes 12-14.

26. Pour l'absence apparente du signe $p r$ dans la graphie donnée par la table d'offrandes susmentionnée Caire CG 23160, voir notre explication supra établissant un parallèle entre $w n$ - $^{`} n p r$... et $w n-p r n \ldots$

27. Les seules occurrences de graphies hiéroglyphiques de iry- 3 pourvues du signe de la maison se trouvent dans une stèle thébaine datée du règne de Taharqa $\left(\mathrm{XXV}^{\mathrm{e}}\right.$ dynastie) et publiée par E. Graefe et W. Wassef, MDAIK, 35 (1979), p. 103-118; voir Hoffmann et Quack, p. 139, n. 94; mais il s'agit dans tous ces exemples de graphies pleines et parfaitement explicites du titre iry- 3 où le signe de la maison 
Graphies abrégées de iry- $3: \square$ (hiéroglyphique); (démotique).

Graphies abrégées de wn-pr:

Historiquement, iry- 3 désigne au moins depuis le Nouvel Empire une fonction purement matérielle liée à toutes sortes d'administrations ou d'institutions, qu'elles soient profanes ou religieuses, tandis que wn-pr n'apparaît qu'à partir de l'époque saïte et se rapporte exclusivement aux temples. Notre interprétation proposée ci-dessus concernant le pCarlsberg 944 permettrait de lier l'origine de ce titre aux cérémonies wn-pr. Ainsi irry-`3, terme sans connotation religieuse marquée, et malgré sa formation archaïque, aurait pu se maintenir en changeant de sens jusqu'en copte, tandis que le titre $w n-p r$, le « pastophore » des textes grecs, disparaissait avec presque tout le vocabulaire de l'ancienne religion ${ }^{28}$.

ne joue qu'un rôle accessoire de déterminatif secondaire, comme le montre son omission dans deux des six occurrences relevées sur cette même stèle, dont la dernière est justement une graphie raccourcie du titre en question.

28. Le copte apd semble cependant avoir perdu le sens de «portier», remplacé par MNOYT. À noter qu'aucun titre religieux pharaonique n'a survécu en copte, à l'exception de оүннв ( $\left.w^{\complement} b\right)$ et 2ONT ( $\mathrm{hm}$ $n t r)$, ce dernier désignant l'ensemble des prêtres païens. L'absence d'un descendant copte de wn-pr n'est donc pas un argument pour réfuter cette lecture du titre démotique du pastophore, versus Hoffmann et Quack, p. 142. 